\title{
DEMENCIA FRONTOTEMPORAL: FRONTERAS DIAGNÓSTICAS
}

\author{
Ma Cruz Pérez Lancho \\ Profesora Encargada de Cátedra. Facultad de Ciencias de la Salud \\ Universidad Pontificia de Salamanca. \\ mcperezla@upsa.es \\ Sergio García Bercianos \\ Psicólogo e Ingeniero Informático. Universidad Pontificia de Salamanca. \\ sergiogarciacorreo@gmail.com
}

Fecha de Recepción: 2 Abril 2018

Fecha de Admisión: 10 Abril 2018

\section{RESUMEN}

La demencia frontotemporal (DFT) tiene menor prevalencia que la enfermedad de Alzheimer (EA) pero su edad de inicio es más temprana, entre los 50 y 60 años. Por esta causa, supone una gran sobrecarga para los cuidadores y una creciente dependencia para los pacientes durante un largo periodo de tiempo. La DFT engloba a una serie de enfermedades neurodegenerativas que afectan a distintas áreas cerebrales y la naturaleza de los síntomas, en cada uno de los cuadros, dependerá de las áreas que estén afectadas en cada fase de la enfermedad. Así pues, se describen una variante conductual, de predominio frontal y tres variantes de afasia progresiva primaria: no fluente, semántica y logopénica, de predominio temporal. Su estudio es relevante en el ámbito de la neuropsicología porque estas demencias afectan en su inicio a las redes neuronales responsables de la cognición y el ajuste social, el lenguaje y el conocimiento semántico del mundo.

La gran variedad de síntomas que presentan, junto a su naturaleza heterogénea, determinan que tanto al inicio de la enfermedad como durante su evolución, la DFT pueda confundirse con la EA o con otras patologías. El objetivo de este trabajo es presentar sus características principales y sus fronteras diagnósticas para proporcionar herramientas que ayuden al diagnóstico diferencial.

La etiología de la DFT está relacionada con alteraciones de proteínas encargadas del mantenimiento del citoesqueleto de las neuronas, con la regulación de la expresión de genes y con el crecimiento celular. Recientemente, nuevos avances en el estudio de los biomarcadores, de la genética y del diagnóstico por neuroimagen de la DFT han permitido clarificar los criterios diagnósticos de los distintos subtipos, están perfilando la frontera con la EA y establecen una relación neuropatológica con síndromes clínicos del espectro del parkinsonismo atípico, la esclerosis lateral amiotrófica y la apraxia del habla. 


\section{DEMENCIA FRONTOTEMPORAL: FRONTERAS DIAGNÓSTICAS}

Palabras clave: demencia frontotemporal; variante conductual; afasia progresiva primaria; enfermedad de Alzheimer; síndromes motores asociados.

\section{ABSTRACT}

Frontotemporal dementia: diagnostic borders.

Frontotemporal dementia (FTD) has a lower prevalence than Alzheimer Disease (AD), but its age of onset takes place earlier, between 50 and 60 years. For this reason, it supposes a great overload for caregivers and an increasing dependence for patients over a long period of time. FTD comprises a set of neurodegenerative diseases that affect different cerebral cortex areas and the nature of symptoms on each clinical syndrome will depend on the areas affected during each of the different stages of the disease. Thus, a behavioral variant, predominantly frontal and three primary progressive aphasia variants, named non-fluent, semantic and logopenic, all of them predominantly temporal, are described. Their study is particularly relevant in the field of neuropsychology because initially these dementias affect the neural networks supporting cognition and social-adjustment, language and semantic knowledge of the world.

The great variety of symptoms along with its heterogenic nature determine that at the onset of the disease and during its evolution, FTD can be confused with AD or other pathologies. The aim of this work is to review its main characteristics and diagnosis boundaries in order to provide useful tools for differential diagnosis.

FTD etiology is related to structural changes in the proteins responsible for maintaining the cytoskeleton of neurons, gene expression regulation and cell growth. Recently, new advances in the study of biomarkers, genetics and neuroimage diagnosis of FTD have made it possible to clarify the diagnostic criteria of the different FTD subtypes, are helping to define the boundaries with AD and are allowing to establish a neuropathological relationship with other clinical syndromes including atypical parkinsonism spectrum, amyotrophic lateral sclerosis and apraxia of speech.

Keywords: frontotemporal dementia; behavioral variant; primary progressive aphasia; Alzheimer disease; associated motor syndromes

\section{INTRODUCCIÓN}

Las demencias frontotemporales (DFT) tienen una prevalencia estimada de 15-22 casos/100.000 habitantes y son la causa más común de demencia en adultos menores de 65 años, siendo la edad de presentación más frecuente entre los 50-60 años (Ratnavalli, Brayne, Dawson y Hodges, 2002). Por este inicio temprano, supone una creciente dependencia para los pacientes y una gran sobrecarga para los cuidadores durante un largo periodo de tiempo. En población no anciana, la prevalencia de la DFT es similar a la de la enfermedad de Alzheimer (Rabinovici y Miller, 2010), mientras que en ancianos ha sido históricamente infravalorada (Rabinovici y Miller, 2010; Warren, Rohrer y Rossor, 2013). Afecta tanto a hombres como mujeres, aunque la variante conductual ha sido descrita mayoritariamente en hombres. Su estudio es muy relevante en el ámbito de la neuropsicología ya que estas demencias afectan inicialmente a las redes neuronales responsables de la cognición y el ajuste social, el lenguaje y el conocimiento semántico del mundo.

Las demencias se estudian fundamentalmente desde tres niveles (Weintraub, 2013). El nivel clínico analiza los síntomas y signos expresados por el paciente, observados por el cuidador y verificados por el clínico. El nivel neuroanatómico localiza la disfunción cerebral mientras que el nivel neuropatológico identifica las anormalidades celulares y moleculares de cada patología. Las investigaciones de estos diversos ámbitos encuentran correlaciones entre la expresión de la enfermedad del tejido neuronal, el síndrome clínico, la funcionalidad de las áreas cerebrales y su conectividad estructural y funcional, las proteinopatías, las alteraciones genéticas y los hallazgos en las pruebas 
de neuroimagen (Pievani, Filippini, van Den Heuvel, Cappa y Frisoni, 2014). Como respuesta a estos desafíos, se proponen nuevos criterios para diagnosticar y clasificar este grupo de demencias. Las nuevas clasificaciones buscan unificar la terminología, más allá de diferencias locales, para que constituyan una base sólida para identificar patologías y compartir información clínica (Lillo y Leyton, 2016). Tal cantidad de áreas de trabajo y especialización origina cierto grado de confusión entre las investigaciones y los clínicos necesitan incorporar los datos provenientes de los estudios de los niveles neuroanatómico y neuropatológicos. El objetivo de este trabajo es presentar la evolución del concepto de la DFT y sus variantes, así como delimitar las actuales fronteras diagnósticas con la enfermedad de Alzheimer, por un lado, y con los síndromes motores asociados por otro, para proporcionar herramientas diagnósticas y de abordaje terapéutico.

\section{SÍNDROMES CLÍNICOS DE LA DEMENCIA FRONTOTEMPORAL}

Arnold Pick, hacia finales del siglo XIX, describe los primeros casos de demencia asociados a problemas de lenguaje y conducta, por atrofia en el lóbulo temporal izquierdo y no debidos a una lesión cerebrovascular (Mesulam et al., 2014). Durante la primera mitad del siglo XX, el estudio de las demencias se centró en separar la enfermedad descrita por Alois Alzheimer de la enfermedad de Pick. En la primera se destacaron los síntomas amnésicos, mientras que en la segunda destacaban los síntomas conductuales y del lenguaje (Lillo y Leyton, 2016; Leyton y Ballard, 2016). La enfermedad de Pick, también llamada demencia frontotemporal, se refirió a un trastorno no homogéneo que incluía a un conjunto de diferentes subtipos clínicos causados por varios procesos patológicos (Perry y Rosen, 2016). El término histórico de enfermedad de Pick se reservó para los casos de DFT con hallazgos neuropatológicos de cuerpos de Pick (Woollacott y Rohrer, 2016).

Desde una perspectiva neuropatológica, Englund et al., (1994) y Neary et al., (1998) sentaron las bases para la clasificación diagnóstica de la DFT bajo los términos de degeneración lobar frontotemporal o degeneración del lóbulo frontotemporal (DLFT) subdividiéndola en tres síndromes clínicos: Demencia frontotemporal, afasia progresiva no fluente (APNF) y demencia semántica (DS). La clasificación actual de la DFT se completa con la descripción de una variante conductual (DFTVC) (Rascovsky et al., 2011). Por lo tanto, el término DFT en la actualidad comprendería tres subsíndromes: DFTVC, DS y APNF. El primero con afectación del área de la conducta y del control ejecutivo y los otros dos con una afectación del lenguaje (Woollacott y Rohrer, 2016).

Hablamos del espectro de la demencia frontotemporal, en primer lugar, porque engloba a varias enfermedades proteínicas, taupatías y ubiquitinopatías, que se agrupan bajo la denominación DLFT (OIney, Spina y Miller., 2017; Perry y Rosen, 2016; Warren et al., 2013). En segundo lugar, porque existe un grado de solapamiento variable entre los síndromes clínicos DFT y otros síndromes relacionados con las enfermedades de la neurona motora (EENM) y el parkinsonismo atípico (van Es et al., 2017; Warren et al., 2013). Finalmente, algunos autores postulan que podría tratarse de una única enfermedad cuyos mecanismos y procesos aún no son del todo comprendidos, y cuya expresión en los diferentes síndromes clínicos varía en función de las regiones de afectación cerebral (Neary et al., 1998; van Es et al., 2017).

El espectro de la DFT también se denomina complejo de Pick (Kertesz y Munoz, 1998) englobando a unas veinte patologías, entre las que se encuentran la enfermedad de cuerpos de Pick, DLFT, APP, degeneración corticobasal, síndrome de degeneración corticobasal, demencia semántica, demencia frontotemporal y demencia por enfermedad de la neurona motora.

Recientemente se sostiene que, aunque a nivel neuropatológico existe cierta correspondencia entre la DFT y la DLFT, ni todos los síndromes con sustrato neuropatológico DLFT son DFT, ni todos los síndromes DFT tienen neuropatología DLFT (Olney et al., 2017; Perry y Rosen, 2016). 


\section{DEMENCIA FRONTOTEMPORAL: FRONTERAS DIAGNÓSTICAS}

\section{AFASIA PROGRESIVA PRIMARIA}

El acuerdo internacional para el diagnóstico de la afasia progresiva primaria (APP) (GornoTempini et al., 2011) sentó las bases para su diagnóstico (ver Figura 1) con algunas consideraciones: incluyen la variante logopénica que antes no era tenida en cuenta. En la DS, excluyen aquellos casos con trastornos agnósicos. Clasifican los síndromes clínicos sin tener en cuenta la enfermedad subyacente, abriendo así la frontera diagnóstica a otras patologías neuronales, como por ejemplo la EA (ver figura 3).

Figura 1: Esquema histórico de la evolución de los criterios diagnósticos de la Afasia Progresiva Primaria

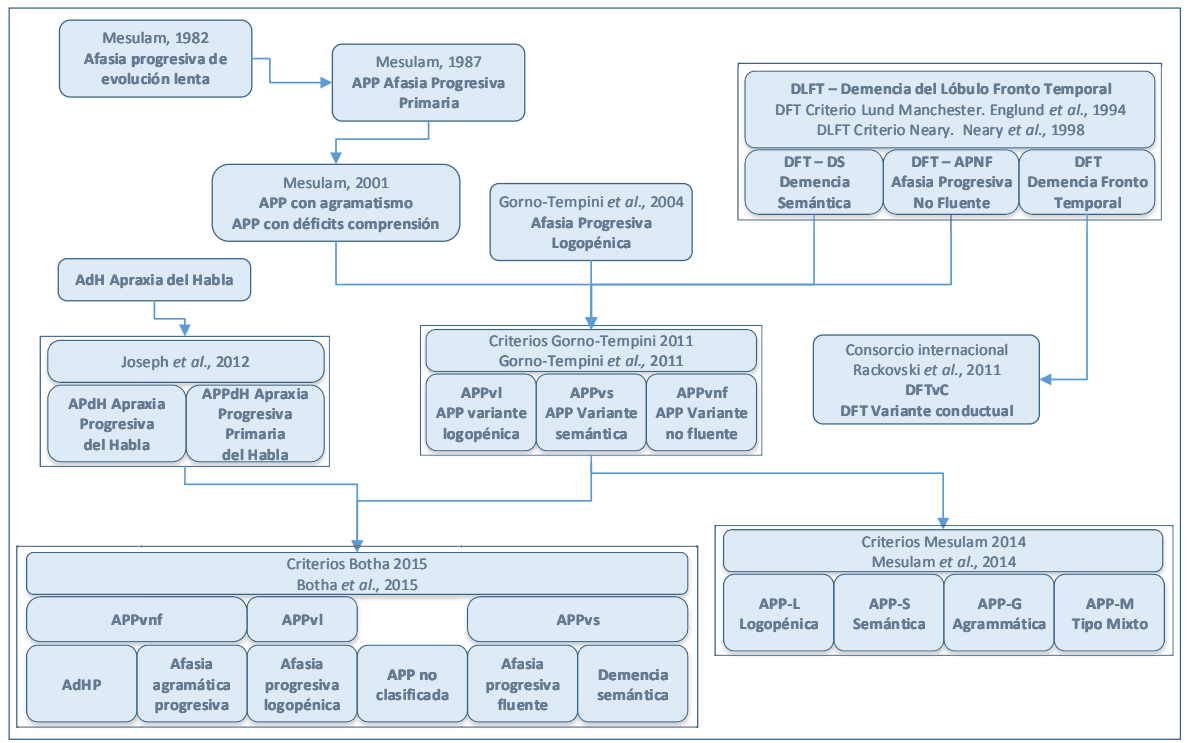

Nota: Adaptado de Leyton y Ballard (2016, p. 13).

De esta forma van surgiendo clasificaciones diagnósticas más ajustadas a los síntomas y al patrón de la enfermedad subyacente, quedando en la actualidad divididas en:

APP variante no fluente o agramática (APPvnF) relacionada con el diagnóstico de APNF (Harciarek y Kertesz, 2011) y que comparte algunas características con la afasia de Broca (Mesulam, 2014).

APP variante semántica (APPvS), también conocida como variante temporal de la DFT (DFTVT), APP fluente (Harciarek y Kertesz, 2011) o como forma no agnósica de la DS (Rogalski y Mesulam, 2007), ya que el diagnóstico de DS incluía a pacientes con agnosia, cuyo fenotipo de enfermedad es inconsistente con el de APP (Mesulam, 2001; Mesulam et al., 2014). Comparte algunas características con la afasia sensorial transcortical (Mesulam, 2014).

APP variante logopénica (APPVL), también conocida como afasia progresiva logopénica (APL) (Gorno Tempini et al., 2004), relacionada con la afasia progresiva mixta (Grossman, 2010) y recientemente considerada como espectro logopénico (Giannini et al., 2017). Algunas clasificaciones la incluirán en la DFT y otras no, debido a que la neuropatología subyacente puede ser de tipo Alzheimer en más de la mitad de los casos. Comparte algunas características con la afasia de conducción 0 la afasia anómica (Mesulam, 2014). 
El trastorno del lenguaje que se manifiesta en pacientes con APP tomará la forma de una variante u otra dependiendo de la localización anatómica de la atrofia cortical (Mesulam et al., 2014).

Esta clasificación de 2011 no está exenta de cierta polémica, pues el deterioro en la repetición de frases, que no formaba parte de la definición original de la logopenia, representa una inconsistencia diagnóstica, además de excluir de la clasificación de la APPvL, a algunos pacientes. Por este motivo, Mesulam (2014) propone unos criterios simplificados para la clasificación de las APP, añadiendo un tipo mixto para aquellos casos que no encajan bien con los criterios de las tres categorías principales de APP (ver figura 1).

Además, se considera como afasia progresiva primaria plus (APP+), a aquel estadio en el desarrollo de la APP en el que los síntomas del lenguaje ya no son la única característica saliente, sino que se afectan otros dominios porque la enfermedad subyacente se ha extendido a redes neuronales adyacentes a las áreas del lenguaje (Mesulam, 2001; Mesulam et al., 2014).

\section{DEMENCIA POR ENFERMEDAD DE ALZHEIMER Y DFT}

Aunque la enfermedad de Alzheimer (EA) constituye la principal causa de las demencias que cursan con un grave deterioro de memoria el término más apropiado es demencia por enfermedad de Alzheimer (DEA). La DEA no solo recoge los síndromes que debutan con trastornos amnésicos, sino que también puede incluir a síndromes en los que predominan la disfunción frontal o parietal (Mesulam et al., 2008). Asimismo, se describen cuadros de EA en los que predominan los problemas en el área del lenguaje y que se vinculan con la logopenia. Originalmente, dicho término fue empleado en el ámbito de la afasia progresiva por Mesulam (1982), para diferenciarlo de las formas no fluentes y describía un trastorno de habla lenta, laboriosa y llena de largas pausas anómicas, probablemente de origen amnésico. Dos décadas más tarde, Gorno-Tempini et al. (2004) acuñan el término de APL como un nuevo subtipo de APP. Esta asociación entre la variante lingüística de la EA y la APL de las demencias frontotemporales se consolida en la actualidad dado que muchos autores consideran que la neuropatología subyacente a la APL es de tipo EA en una mayoría de los casos (Giannini et al., 2017; Gorno-Tempini et al., 2011; Leyton y Ballard, 2016; Perry y Rosen, 2016; Woollacott y Rohrer, 2016) aunque se presente como una forma atípica de EA (Mesulam et al, 2014; Pievani et al., 2014)

\section{SÍNDROMES MOTORES ASOCIADOS A LA DFT}

Uno de los problemas principales a la hora de clarificar la descripción de los síndromes clínicos de DFT y APP es que existe un grado de solapamiento entre ambos, y además entre éstos y dos grupos de patologías más, el parkinsonismo atípico y el espectro de la esclerosis lateral amiotrófica (Kertesz y Munoz, 2004; Warren et al., 2013).

\section{Síndromes asociados al parkinsonismo atípico}

Los más característicos son los síndromes de parálisis supranuclear progresiva (SPSP) y el síndrome de degeneración cortico basal (SCB). El SPSP suele presentar parálisis de los movimientos sacádicos verticales y a una marcada inestabilidad postural, con caídas frecuentes, mientras que el SCB suele presentar parkinsonismo asimétrico sin afectación de la deambulación, apraxia ideomotora, mioclonía y distonía (Tapia-Núñez y Chana-Cuevas, 2004).

Kertesz, Blair, McMonagle y Munoz (2007) describieron el solapamiento entre estos síndromes, indicando que los pacientes con APP pueden desarrollar con el tiempo un segundo e incluso un tercer diagnóstico que puede incluir DFTVC, SPSP, SCB e incluso enfermedades de la neurona motora (EENM). 


\section{DEMENCIA FRONTOTEMPORAL: FRONTERAS DIAGNÓSTICAS}

\section{El espectro de la esclerosis lateral amiotrófica.}

El espectro de la esclerosis lateral amiotrófica (EELA) también llamado de las EENM, se caracteriza por una pérdida progresiva de las neuronas motoras en el cerebro y/o en la médula espinal. Consideradas tradicionalmente como enfermedades neuromusculares, su comorbilidad con las demencias, especialmente la DFT, está cambiando la forma en que son abordadas en la clínica y en la investigación (van Es et al., 2017).

El EELA engloba una serie de síndromes que podrían formar un continuo fenotípico de la misma enfermedad en cuyos extremos se situarían la esclerosis lateral amiotrófica (ELA) y la DFT (incluyendo la APP) (van Es et al., 2017), y donde encontraríamos un grado variable de solapamiento en los estadios intermedios (ver figura 2).

Figura 2: Continuo espectro esclerosis lateral amiotrófica - demencia frontotemporal

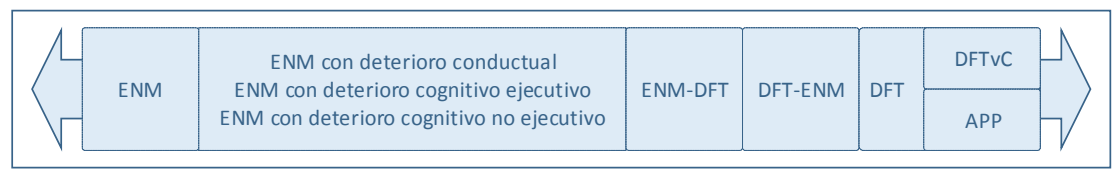

Nota: ENM: enfermedad de la neurona motora. DFT: demencia frontotemporal. ENM-DFT: enfermedad de la neurona motora con diagnóstico posterior de DFT. DFT-ENM: diagnóstico de DFT con diagnóstico posterior de enfermedad de la neurona motora. DFTvC: demencia frontotemporal variante conductual. APP: afasia progresiva primaria. Adaptado de van Es et al. (2017, p.2).

\section{APRAXIA DEL HABLA}

La apraxia del habla $(\mathrm{AdH})$ es un trastorno en la planificación y/o programación motora del habla que cursa con un comienzo insidioso hasta llegar generalmente al mutismo. Debido su reciente diagnóstico y a que tradicionalmente había sido incluida en la APP o confundida con disartria, no ha sido aceptada como una enfermedad neurodegenerativa per se. De hecho, el diagnóstico de AdH por sí solo no es causa suficiente para el diagnóstico de afasia, porque se conserva la función del lenguaje (Josephs et al., 2012).

Duffy y Josephs (2012), utilizan el término apraxia progresiva del habla (APdH) para diferenciarla de la AdH no progresiva. Josephs et al. (2012) acuñan además un nuevo término para definir los casos con enfermedad neurodegenerativa y AdH como único síntoma saliente, la apraxia progresiva primaria del habla (APPdH) (ver figura 1).

El diagnóstico clínico de APdH se puede solapar con otros, como la APP, SPSP, SCB o las EENM (Duffy, Strand y Josephs, 2014). El parkinsonismo suele aparecer en los estudios Iongitudinales de la $\mathrm{APdH}$, y un pequeño grupo desarrolla un síndrome similar al SPSP sin inestabilidad postural (Poole, Brodtmann, Darby y Vogel, 2017).

\section{CONSIDERACIONES EN EL ÁMBITO DE LOS SÍNDROMES CLíNICOS}

Para finalizar, es preciso añadir algunas consideraciones más sobre las patologías asociadas a la DFT. Recientemente, Botha et al. (2015) han unificado los criterios para la APP en una nueva clasificación diagnóstica que incluye la APdH, junto a una reformulación de los criterios de los diferentes subtipos de APP.

En algunos pacientes con superposición de síndromes demenciales y síndromes motores, los síntomas conductuales y cognitivos pueden aparecer antes, después, o coexistir con los síntomas motores, lo que en la práctica clínica dificulta el diagnóstico (Woollacott y Rohrer, 2016). Ante el solapamiento de los síndromes, el orden del diagnóstico tendrá importantes repercusiones en el tratamiento y en el enfermo y su entorno. Un diagnóstico de una enfermedad de la neurona motora 
(ENM) que es seguido de un diagnóstico de DFT (ENM-DFT) se diferenciará de un diagnóstico de DFT que es seguido en el tiempo de un diagnóstico de una ENM (DFT-ENM) (ver figura 2), por el distinto recorrido, estudio y tratamiento de cada caso en particular. La diferencia radica en el síndrome 0 enfermedad que es diagnosticada en primer lugar.

Como síntesis, en la figura 3 se presenta la relación entre la DFT con los síndromes motores.

Figura 3: Indicadores de solapamiento entre los principales síndromes clínicos

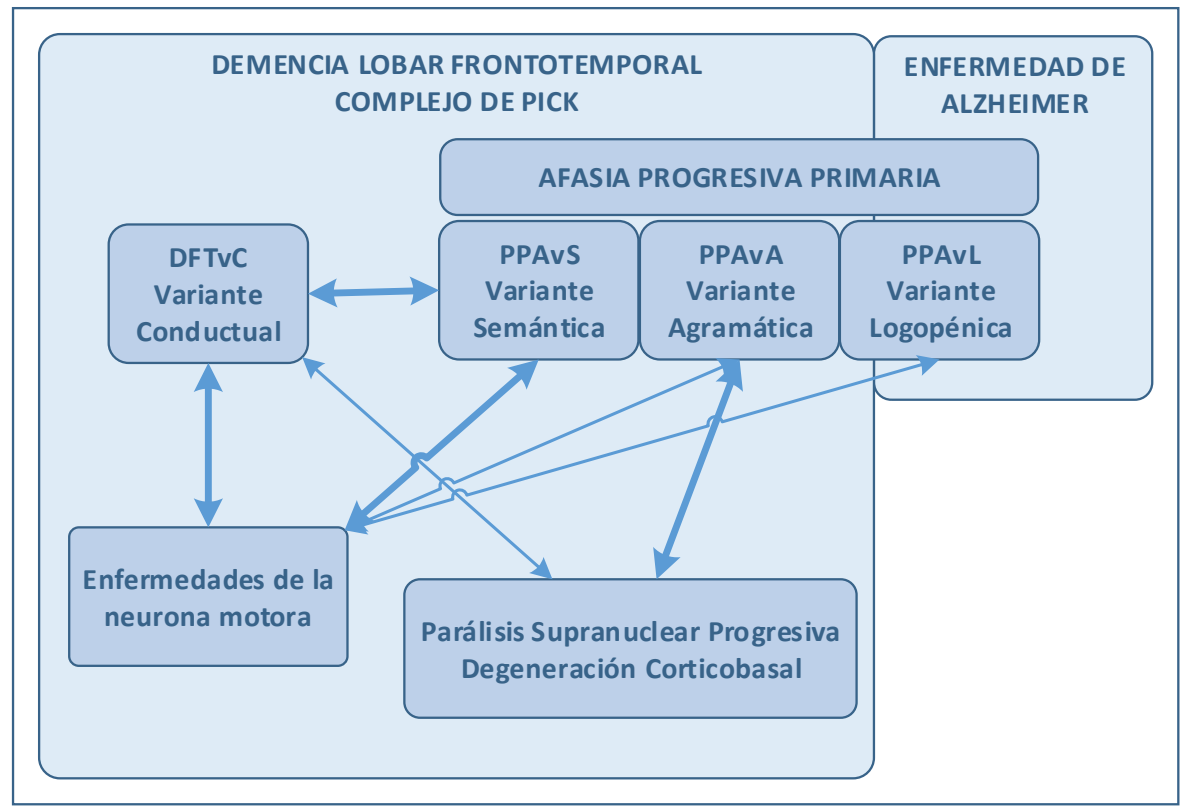

Nota: El grosor de las líneas indica el grado relativo de solapamiento entre los diferentes síndromes. Adaptado de Harciarek y Kertesz (2011, p.273).

\section{CONCLUSIONES}

Las demencias frontotemporales son la causa más común de deterioro cognitivo progresivo en adultos menores de 65 años (Ratnavalli et al., 2002). Su estudio es relevante porque debutan con alteraciones de la cognición y el ajuste social, del lenguaje o del conocimiento semántico. Los pacientes deben afrontar una dependencia funcional creciente durante décadas con la consiguiente sobrecarga para los cuidadores. Progresivamente, la degeneración neuronal se extiende por otras áreas cerebrales y sus síntomas pueden solaparse con otros síndromes como la demencia tipo Alzheimer, la apraxia del habla u otras enfermedades motoras que complican aún más el manejo del paciente.

La investigación sobre DFT ha ido correlacionado los síndromes clínicos con las funciones cerebrales, las proteinopatías subyacentes, los estudios genéticos y las pruebas de neuroimagen (Pievani et al., 2014). Este hecho ha obligado a proponer sucesivas clasificaciones y a vincular las DFT con otras patologías que comparten el tipo de proteínas o genes alterados (Lillo y Leyton, 2016). El presente trabajo se ha centrado en presentar los diferentes criterios diagnósticos de la DFT con sus variantes y en delimitar sus fronteras diagnósticas con otros síndromes asociados. 


\section{DEMENCIA FRONTOTEMPORAL: FRONTERAS DIAGNÓSTICAS}

Después de un análisis del estado del arte, concluimos que las DFT en la actualidad pueden clasificarse en una variante conductual DFTVC, de predominio frontal y tres variantes de lenguaje, de predominio temporal: la APP variante no fluente 0 agramática, APP variante semántica, APP fluente y APP variante logopénica.

En la actualidad la demencia tipo Alzheimer no solo incluye a los síndromes que debutan con trastornos amnésicos, sino que también engloba a síndromes en los que predominan la disfunción frontal, parietal o problemas de lenguaje (Mesulam et al., 2008). La EA de variante lingüística se solapa de forma importante con la variante logopénica de la DFT y comparte con ella las mismas alteraciones proteínicas.

La DFT también se relaciona con los síndromes motores por compartir hallazgos neuropatológicos y proteinopatías comunes. En primer lugar, la DFT se asocia con síndromes de parkinsonismo atípico: el síndrome de parálisis supranuclear progresiva y la degeneración corticobasal. Por otro lado, el espectro de la ELA es un conjunto de enfermedades clásicamente consideradas como enfermedades neuromusculares, y que sin embargo muestran comorbilidad con las demencias frontotemporales, conformando un continuo ELA-DFT (van Es et al., 2017).

Por último, se ha descrito una forma progresiva de apraxia del habla (Duffy y Josephs, 2012) que, afectando a la programación de los movimientos del habla, se sitúa en la frontera funcional con las afasias progresivas primarias no fluentes.

Aunque se han realizado numerosos avances en la descripción de las DFT, consideramos que la delimitación de este panorama diagnóstico está lejos de considerarse finalizada. Persisten ciertas inconsistencias que exigen la consideración de tipos mixtos de APP (Mesulam et al., 2014) y de una forma de afasia progresiva multidominio, APP plus, para clasificar algunos casos. Es por ello que la investigación clínica debe perseverar en la revisión de sus criterios diagnósticos incorporando las aportaciones del estudio de biomarcadores, en un esfuerzo por comprender los mecanismos neurodegenerativos de las demencias.

\section{REFERENCIAS BIBLIOGRÁFICAS}

Botha, H., Duffy, J. R., Whitwell, J. L., Strand, E. A., Machulda, M. M., Schwarz, C. G., ... Josephs, K. A. (2015). Classification and clinicoradiologic features of primary progressive aphasia (PPA) and apraxia of speech. Cortex, 69, 220-236. doi: 10.1016/j.cortex.2015.05.013.

Duffy, J. R., y Josephs, K. A. (2012). The diagnosis and understanding of apraxia of speech: Why including neurodegenerative etiologies may be important. Journal of Speech, Language, and Hearing Research, 55(5), S1518-S1522. doi: 10.1044/1092-4388(2012/11-0309).

Duffy, J. R., Strand, E. A., y Josephs, K. A. (2014). Motor speech disorders associated with primary progressive aphasia. Aphasiology, 28(8-9), 1004-1017. doi: 10.1080/02687038.2013.869307.

Englund, B., Brun, A., Gustafson, L., Passant, U., Mann, D., Neary, D., y Snowden, J. S. (1994). Clinical and neuropathological criteria for frontotemporal dementia. Journal of Neurology, Neurosurgery and Psychiatry, 57(4), 416-8. doi: 10.1136/jnnp.57.4.416.

Giannini, L. A. A., Irwin, D. J., McMillan, C. T., Ash, S., Rascovsky, K., Wolk, D. A., ... Grossman, M. (2017). Clinical marker for Alzheimer disease pathology in logopenic primary progressive aphasia. Neurology, 88(24), 2276-2284. doi: 10.1212/WNL.0000000000004034.

Gorno Tempini, M. L., Dronkers, N. F., Rankin, K. P., Ogar, J. M., Phengrasamy, L., Rosen, H. J., ... Miller, B. L. (2004). Cognition and anatomy in three variants of primary progressive aphasia. Annals of Neurology, 55(3), 335-346. doi: 10.1002/ana.10825.

Gorno-Tempini, M. L., Hillis, A. E., Weintraub, S., Kertesz, A., Mendez, M., Cappa, S. F., ... Grossman, M. (2011). Classification of primary progressive aphasia and its variants. Neurology, 76(11), 1006-1014. doi:10.1212/WNL.0b013e31821103e6. 
Grossman, M. (2010). Primary progressive aphasia: Clinicopathological correlations. Nature Reviews Neurology, 6(2), 88-97. doi: 10.1038/nrneurol.2009.216.

Harciarek, M., y Kertesz, A. (2011). Primary progressive aphasias and their contribution to the contemporary knowledge about the brain-language relationship. Neuropsychology Review, 21(3), 271-287. doi: 10.1007/s11065-011-9175-9.

Josephs, K. A., Duffy, J. R., Strand, E. A., Machulda, M. M., Senjem, M. L., Master, A. V., ... Whitwell, J. L. (2012). Characterizing a neurodegenerative syndrome: Primary progressive apraxia of speech. Brain, 135(5), 1522-1536. doi: 10.1093/brain/aws032.

Kertesz, A., Blair, M., McMonagle, P., y Munoz, D. G. (2007). The diagnosis and course of frontotemporal dementia. Alzheimer Disease \& Associated Disorders, 21(2), 155-163. doi: 10.1097/wad.0b013e31806547eb.

Kertesz, A., y Munoz, D. (1998). Pick's disease, frontotemporal dementia, and Pick complex: Emerging concepts. Archives of Neurology, 55(3), 302-304. doi: 10.1001/archneur.55.3.302.

Kertesz, A., y Munoz, D. (2004). Relationship between frontotemporal dementia and corticobasal degeneration/progressive supranuclear palsy. Dementia and Geriatric Cognitive Disorders, 17(4), 282-286. doi: 10.1159/000077155.

Mesulam, M. M. (1982). Slowly progressive aphasia without generalized dementia. Annals of Neurology, 11(6), 592-598. doi: 10.1002/ana.410110607.

Mesulam, M. M. (2001). Primary progressive aphasia. Annals of Neurology, 49(4), 425-432. doi: 10.1002/ana.91.

Mesulam, M. M. (2014). Primary Progressive Aphasia. En B. Dickerson y A. Atri (Eds.), Dementia: Comprehensive Principles and Practices (pp. 198-207). New York: Oxford University Press.

Mesulam, M. M. (2016). Primary Progressive Aphasia and the Left Hemisphere Language Network. Dementia and Neurocognitive Disorders, 15(4), 93-102. doi:10.12779/dnd.2016.15.4.93.

Mesulam, M. M., Rogalski, E. J., Wieneke, C., Hurley, R. S., Geula, C., Bigio, E. H., ... Weintraub, S. (2014). Primary progressive aphasia and the evolving neurology of the language network. Nature Reviews Neurology, 10(10), 554-569. doi: 10.1038/nrneurol.2014.159.

Mesulam, M. M., Wicklund, A., Johnson, N., Rogalski, E., Léger, G. C., Rademaker, A., Weintraub, S., y Bigio, E. H. (2008). Alzheimer and frontotemporal pathology in subsets of primary progressive aphasia. Annals of Neurology, 63(6), 709-719. doi: 10.1002/ana.21388.

Neary, D., Snowden, J. S., Gustafson, L., Passant, U., Stuss, D., Black, S., ... Benson, D. F. (1998). Frontotemporal lobar degeneration: A consensus on clinical diagnostic criteria. Neurology, 51(6), 1546-1554. doi: 10.1212/wnl.51.6.1546.

Leyton, C. E., y Ballard, K. J. (2016). Primary progressive aphasia: Conceptual evolution and challenges. Neuroscience and Neuroeconomics, 5, 9-18. doi: 10.2147/NAN.S102848.

Lillo, P., y Leyton, C. (2016). Demencia frontotemporal, cómo ha resurgido su diagnóstico. Revista Médica Clínica Las Condes, 27(3), 309-318. doi: 10.1016/j.rmclc.2016.06.005.

Olney, N. T., Spina, S., y Miller, B. L. (2017). Frontotemporal dementia. Neurologic Clinics, 35(2), 339-374. doi: 10.1016/j.ncl.2017.01.008

Perry, D. C., y Rosen, H. J. (2016). Frontotemporal dementia. En M. D. Geschwind y C. R. Belkoura (Eds.), Non-Alzheimer's and Atypical Dementia (pp. 49-63). Oxford: John Wiley \& Sons.

Pievani, M., Filippini, N., van Den Heuvel, M. P., Cappa, S. F., y Frisoni, G. B. (2014). Brain connectivity in neurodegenerative diseases from phenotype to proteinopathy. Nature Reviews: Neurology. Publicación anticipada en línea. doi: 10.1038/nrneurol.2014.178.

Poole, M. L., Brodtmann, A., Darby, D., y Vogel, A. P. (2017). Motor Speech Phenotypes of Frontotemporal Dementia, Primary Progressive Aphasia, and Progressive Apraxia of Speech. 


\section{DEMENCIA FRONTOTEMPORAL: FRONTERAS DIAGNÓSTICAS}

Journal of Speech, Language, and Hearing Research, 60(4), 897-911. doi: 10.1044/2016_JSLHR-S-16-0140.

Rabinovici, G. D., y Miller, B. L. (2010). Frontotemporal lobar degeneration. CNS drugs, 24(5), 375398. doi: 10.2165/11533100-000000000-00000.

Rascovsky, K., Hodges, J. R., Knopman, D., Mendez, M. F., Kramer, J. H., Neuhaus, J., ... Miller, B. L. (2011). Sensitivity of revised diagnostic criteria for the behavioural variant of frontotemporal dementia. Brain, 134(9), 2456-2477. doi: 10.1093/brain/awr179.

Ratnavalli, E., Brayne, C., Dawson, K., y Hodges, J. R. (2002). The prevalence of frontotemporal dementia. Neurology, 58(11), 1615-1621. doi: 10.1212/WNL.58.11.1615.

Rogalski, E., y Mesulam, M. M. (2007). An update on primary progressive aphasia. Current Neurology and Neuroscience Reports, 7(5), 388-392. doi: 10.1007/s11910-007-0060-0.

Tapia-Núñez, J., y Chana-Cuevas, P. (2004). Diagnóstico de la enfermedad de Parkinson. Revista de Neurología, 38(1), 61-67. Recuperado de: https://www.ncbi.nlm.nih.gov/ pubmed/14730494.

van Es, M. A., Hardiman, 0., Chio, A., Al-Chalabi, A., Pasterkamp, R. J., Veldink, J. H., y van den Berg, L. H. (2017). Amyotrophic lateral sclerosis. The Lancet. Publicación anticipada en línea. doi: 10.1016/S0140-6736(17)31287-4.

Warren, J. D., Rohrer, J. D., y Rossor, M. N. (2013). Frontotemporal dementia. BMJ, 347. doi: 10.1136/bmj.f4827

Weintraub, S. (2013, 1 de mayo). The ABCs of PPA for SLPs: Clinical Attributes, Biology and Care of Primary Progressive Aphasia [Seminario Web]. Cognitive Neurology and Alzheimer's Disease Center. Recuperado de: http://files.ctctcdn.com/e5276918001/ 02e419b8-07be-40e7-b9bbccbd8304692f.pdf

Woollacott, I. 0., y Rohrer, J. D. (2016). The clinical spectrum of sporadic and familial forms of frontotemporal dementia. Journal of Neurochemistry, 138(S1), 6-31. doi: 10.1111/jnc.13654. 